\title{
ENUMERATION OF HAMILTONIAN CYCLES AND PATHS IN A GRAPH
}

\author{
C. J. LIU
}

(Communicated by Andrew Odlyzko)

\begin{abstract}
First, we show that the determinant of a given matrix can be expanded by its principal minors together with a set of arbitrary parameters. The enumeration of Hamiltonian cycles and paths in a graph is then carried out by an algebraic method. Three types of nonalgebraic representation are formulated. The first type is given in terms of the determinant and permanent of a parametrized adjacent matrix. The second type is presented by a determinantal function of multivariables, each variable having domain $\{0,1\}$. Formulas of the third type are expressed by spanning trees of subgraphs. When applying the formulas to a complete multipartite graph, one can easily find the results.
\end{abstract}

\section{INTRODUCTION}

Let $\mathbf{A}=\left(a_{i j}\right)$ be the adjacency matrix of graph $G$. The corresponding Kirchhoff matrix $\mathbf{K}=\left(k_{i j}\right)$ is obtained from $\mathbf{A}$ by replacing in $-\mathbf{A}$ each diagonal entry by the degree of its corresponding vertex; i.e., the $i$ th diagonal entry is identified with the degree of the $i$ th vertex. It is well known that

$$
\operatorname{det} \mathbf{K}(i \mid i)=\text { number of spanning trees of } G, \quad i=1, \ldots, n
$$

where $\mathbf{K}(i \mid i)$ is the $i$ th principal submatrix of $\mathbf{K}$.

Let $C_{i(j)}$ be the set of graphs obtained from $G$ by attaching edge $\left(v_{i} v_{j}\right)$ to each spanning tree of $G$. Denote by $C_{i}=\bigcup_{j} C_{i(j)}$. It is obvious that the collection of Hamiltonian cycles is a subset of $C_{i}$. Note that the cardinality of $C_{i}$ is $k_{i i} \operatorname{det} \mathbf{K}(i \mid i)$. Let $\widehat{X}=\left\{\hat{x}_{1}, \ldots, \hat{x}_{n}\right\}$. Define multiplication for the elements of $\widehat{X}$ by

$$
\hat{x}_{i} \hat{x}_{j}=\hat{x}_{j} \hat{x}_{i}, \quad \hat{x}_{i}^{2}=0, \quad i, j=1, \ldots, n .
$$

Let $\hat{k}_{i j}=k_{i j} \hat{x}_{j}$ and $\hat{k}_{i i}=-\sum_{j \neq i} \hat{k}_{i j}$. Then the number of Hamiltonian cycles

Received by the editors October 23, 1989.

1980 Mathematics Subject Classification (1985 Revision). Primary 05C38; Secondary 05A15, $15 \mathrm{~A} 15,15 \mathrm{~A} 18$.

This work was partially supported by Marquette University Sabbatical Plan (Spring, 1987). 
$H_{c}$ is given by the relation [1]

$$
\left(\prod_{j=1}^{n} \hat{x}_{j}\right) H_{c}=\frac{1}{2} \hat{k}_{i i} \operatorname{det} \widehat{\mathbf{K}}(i \mid i), \quad i=1, \ldots, n .
$$

The task here is to express (3) in a form free of any $\hat{x}_{i}, i=1, \ldots, n$. The result also leads to the resolution of enumeration of Hamiltonian paths in a graph.

It is well known that the enumeration of Hamiltonian cycles and paths in a complete graph $K_{n}$ and in a complete bipartite graph $K_{n_{1} n_{2}}$ can only be found from first combinatorial principles [2]. One wonders if there exists a formula which can be used very efficiently to produce $K_{n}$ and $K_{n_{1} n_{2}}$. Recently, using Lagrangian methods, Goulden and Jackson have shown that $H_{c}$ can be expressed in terms of the determinant and permanent of the adjacency matrix [3]. However, the formula of Goulden and Jackson determines neither $K_{n}$ nor $K_{n_{1} n_{2}}$ effectively. In this paper, using an algebraic method, we parametrize the adjacency matrix. The resulting formula also involves the determinant and permanent, but it can easily be applied to $K_{n}$ and $K_{n_{1} n_{2}}$. In addition, we eliminate the permanent from $H_{c}$ and show that $H_{c}$ can be represented by a determinantal function of multivariables, each variable with domain $\{0,1\}$. Furthermore, we show that $H_{c}$ can be written by number of spanning trees of subgraphs. Finally, we apply the formulas to a complete multipartite $K_{n_{1} \cdots n_{p}}$.

The conditions $a_{i j}=a_{j i}, i, j=1, \ldots, n$, are not required in this paper. All formulas can be extended to a digraph simply by multiplying $H_{c}$ by 2 .

\section{MAIN THEOREM}

Let $\mathbf{B}=\left(b_{i j}\right)$ be an $n \times n$ matrix. Let $\mathbf{n}=\{1, \ldots, n\}$. Using the properties of $(2)$, it is readily seen that

\section{Lemma 1.}

$$
\prod_{i \in \mathbf{n}}\left(\sum_{j \in \mathbf{n}} b_{i j} \hat{x}_{j}\right)=\left(\prod_{i \in \mathbf{n}} \hat{x}_{i}\right) \operatorname{per} \mathbf{B}
$$

where per $\mathbf{B}$ is the permanent of $\mathbf{B}$.

Let $\hat{Y}=\left\{\hat{y}_{1}, \ldots, \hat{y}_{n}\right\}$. Define multiplication for the elements of $\hat{Y}$ by

$$
\hat{y}_{i} \hat{y}_{j}+\hat{y}_{j} \hat{y}_{i}=0, \quad i, j=1, \ldots, n \text {. }
$$

Then, it follows that

Lemma 2.

$$
\prod_{i \in \mathbf{n}}\left(\sum_{j \in \mathbf{n}} b_{i j} \hat{y}_{j}\right)=\left(\prod_{i \in \mathbf{n}} \hat{y}_{i}\right) \operatorname{det} \mathbf{B} .
$$


Note that all basic properties of determinants are direct consequences of Lemma 2 .

Write

$$
\sum_{j \in \mathbf{n}} b_{i j} \hat{y}_{j}=\sum_{j \in \mathbf{n}} b_{i j}^{(\lambda)} \hat{y}_{j}+\left(b_{i i}-\lambda_{i}\right) \hat{y}_{i}
$$

where

$$
b_{i i}^{(\lambda)}=\lambda_{i}, \quad b_{i j}^{(\lambda)}=b_{i j}, \quad i \neq j .
$$

Let $\mathbf{B}^{(\lambda)}=\left(b_{i j}^{(\lambda)}\right)$. By (6) and (7), it is straightforward to show the following result :

\section{Theorem 1.}

$$
\operatorname{det} \mathbf{B}=\sum_{l=0}^{n} \sum_{I_{l} \subseteq \mathbf{n}} \prod_{i \in I_{l}}\left(b_{i i}-\lambda_{i}\right) \operatorname{det} \mathbf{B}^{(\lambda)}\left(I_{l} \mid I_{l}\right),
$$

where $I_{l}=\left\{i_{1}, \ldots, i_{l}\right\}$ and $\mathbf{B}^{(\lambda)}\left(I_{l} \mid I_{l}\right)$ is the principal submatrix obtained from $\mathbf{B}^{(\lambda)}$ by deleting its $i_{1}, \ldots, i_{l}$ rows and columns.

Remark. Let $\mathbf{M}$ be an $n \times n$ matrix. The convention $\mathbf{M}(\mathbf{n} \mid \mathbf{n})=1$ has been used in (9) and hereafter.

Before proceeding with our discussion, we pause to note that Theorem 1 yields immediately a fundamental formula which can be used to compute the coefficients of a characteristic polynomial [4]:

Corollary. Write $\operatorname{det}(\mathbf{B}-x \mathbf{I})=\sum_{l=0}^{n}(-1)^{l} b_{l} x^{l}$. Then

$$
b_{l}=\sum_{I_{l} \subseteq \mathbf{n}} \operatorname{det} \mathbf{B}\left(I_{l} \mid I_{l}\right) \text {. }
$$

Let

where

$$
\mathbf{K}\left(t, t_{1}, \ldots, t_{n}\right)=\left(\begin{array}{cccc}
D_{1} t & -a_{12} t_{2} & \cdots & -a_{1 n} t_{n} \\
-a_{21} t_{1} & D_{2} t & \cdots & -a_{2 n} t_{n} \\
\vdots & \vdots & \vdots & \vdots \\
-a_{n 1} t_{1} & -a_{n 2} t_{2} & \cdots & D_{n} t
\end{array}\right)
$$

Set

$$
D_{i}=\sum_{j \in \mathbf{n}} a_{i j} t_{j}, \quad i=1, \ldots, n
$$

$$
D\left(t_{1}, \ldots, t_{n}\right)=\left.\frac{\partial}{\partial t} \operatorname{det} \mathbf{K}\left(t, t_{1}, \ldots, t_{n}\right)\right|_{t=1} .
$$

Then

$$
D\left(t_{1}, \ldots, t_{n}\right)=\sum_{i \in \mathbf{n}} D_{i} \operatorname{det} \mathbf{K}\left(t=1, t_{1}, \ldots, t_{n} ; i \mid i\right),
$$

where $\mathbf{K}\left(t=1, t_{1}, \ldots, t_{n} ; i \mid i\right)$ is the $i$ th principal submatrix of $\mathbf{K}(t=1$, $\left.t_{1}, \ldots, t_{n}\right)$. 
Theorem 1 leads to

$$
\operatorname{det} \mathbf{K}\left(t, t_{1}, \ldots, t_{n}\right)=\sum_{I \subseteq \mathbf{n}}(-1)^{|I|} t^{n-|I|} \prod_{i \in I} t_{i} \prod_{j \in I}\left(D_{j}+\lambda_{j} t_{j}\right) \operatorname{det} \mathbf{A}^{(\lambda t)}(\bar{I} \mid \bar{I})
$$

Note that

(15) $\operatorname{det} \mathbf{K}\left(t=1, t_{1}, \ldots, t_{n}\right)=\sum_{I \subseteq \mathbf{n}}(-1)^{|I|} \prod_{i \in I} t_{i} \prod_{j \in I}\left(D_{j}+\lambda_{j} t_{j}\right) \operatorname{det} \mathbf{A}^{(\lambda)}(\bar{I} \mid \bar{I})=0$.

It follows from (13), (14), and (15) that

$$
D\left(t_{1}, \ldots, t_{n}\right)=\sum_{I \subseteq \mathbf{n}}(-1)^{|I|-1}|I| \prod_{i \in I} t_{i} \prod_{j \in I}\left(D_{j}+\lambda_{j} t_{j}\right) \operatorname{det} \mathbf{A}^{(\lambda)}(\bar{I} \mid \bar{I}) .
$$

Let $t_{i}=\hat{x}_{i}, i=1, \ldots, n$. Lemma 1 leads to

$$
D\left(\hat{x}_{1}, \ldots, \hat{x}_{n}\right)=\prod_{i \in \mathbf{n}} \hat{x}_{i} \sum_{I \subseteq \mathbf{n}}(-1)^{|I|-1}|I| \operatorname{per} \mathbf{A}^{(\lambda)}(I \mid I) \operatorname{det} \mathbf{A}^{(\lambda)}(\bar{I} \mid \bar{I}) .
$$

By (3), (13), and (17), we have the following result:

\section{Theorem 2.}

$$
H_{c}=\frac{1}{2 n} \sum_{l=1}^{n} l(-1)^{l-1} A_{l}^{(\lambda)}
$$

where

$$
A_{l}^{(\lambda)}=\sum_{I_{l} \subseteq \mathbf{n}} \operatorname{per} \mathbf{A}^{(\lambda)}\left(I_{l} \mid I_{l}\right) \operatorname{det} \mathbf{A}^{(\lambda)}\left(\bar{I}_{l} \mid \bar{I}_{l}\right),\left|I_{l}\right|=l .
$$

It is worth noting that $A_{l}^{(\lambda)}$ of (19) is similar to the coefficients $b_{l}$ of the characteristic polynomial of $(10)$. It is well known in graph theory that the coefficients $b_{l}$ can be expressed as a sum over certain subgraphs. It is interesting to see whether $A_{l}^{0}, \lambda=0$, have the structural properties of a graph.

We may call (18) a parametric representation of $H_{c}$. In computation, the parameter $\lambda_{i}$ plays very important roles. The choice of the parameter usually depends on the properties of the given graph. For a complete graph $K_{n}$, let $\lambda_{i}=1, i=1, \ldots, n$. It follows from (19) that

$$
A_{l}^{(1)}= \begin{cases}n !, & \text { if } l=1 \\ 0, & \text { otherwise. }\end{cases}
$$

By (18)

$$
H_{c}=\frac{1}{2}(n-1) !
$$

For a complete bipartite graph $K_{n_{1} n_{2}}$, let $\lambda_{i}=0, i=1, \ldots, n$. By (19),

$$
A_{l}^{0}= \begin{cases}-n_{1} ! n_{2} ! \delta_{n_{1} n_{2}}, & \text { if } l=2 \\ 0, & \text { otherwise }\end{cases}
$$


Theorem 2 leads to

$$
H_{c}=\frac{1}{n_{1}+n_{2}} n_{1} ! n_{2} ! \delta_{n_{1} n_{2}} .
$$

Now, we consider an asymmetrical approach. Theorem 1 leads to

$$
\begin{aligned}
\operatorname{det} \mathbf{K} & \left(t=1, t_{1}, \ldots, t_{n} ; l \mid l\right) \\
= & \sum_{I \subseteq \mathbf{n}-\{l\}}(-1)^{|I|} \prod_{i \in I} t_{i} \prod_{j \in I}\left(D_{j}+\lambda_{j} t_{j}\right) \operatorname{det} \mathbf{A}^{(\lambda)}(\bar{I} \cup\{l\} \mid \bar{I} \cup\{l\}) .
\end{aligned}
$$

Let $t_{i}=\hat{x}_{i}, i=1, \ldots, n$. Lemma 1 yields

$$
\begin{aligned}
\left(\sum_{i \in \mathbf{n}} a_{l_{i}} \hat{x}_{i}\right) & \operatorname{det} \mathbf{K}\left(t=1, \hat{x}_{1}, \ldots, \hat{x}_{n} ; l \mid l\right) \\
& =\left(\prod_{i \in \mathbf{n}} \hat{x}_{i}\right) \sum_{I \subseteq \mathbf{n}-\{l\}}(-1)^{|I|} \operatorname{per} \mathbf{A}^{(\lambda)}(I \mid I) \operatorname{det} \mathbf{A}^{(\lambda)}(\bar{I} \cup\{l\} \mid \bar{I} \cup\{l\}) .
\end{aligned}
$$

By (3) and (25) we have the following asymmetrical result:

Theorem 3.

$$
H_{c}=\frac{1}{2} \sum_{I \subseteq \mathbf{n}-\{l\}}(-1)^{|I|} \operatorname{per} \mathbf{A}^{(\lambda)}(I \mid I) \operatorname{det} \mathbf{A}^{(\lambda)}(\bar{I} \cup\{l\} \mid \bar{I} \cup\{l\})
$$

which reduces to Goulden-Jackson's formula when $\lambda_{i}=0, i=1, \ldots, n$ [4].

Note that Theorem 3 fails to provide the simple relations of (20) or (22) when the graph $K_{n}$ or $K_{n_{1} n_{2}}$ is treated.

In what follows, we shall formulate the enumeration of the Hamiltonian cycle in an alternative setting to the one (Theorem 2 or 3 ) that involves permanents.

It follows from (15) that

(27) $\operatorname{det} \mathbf{K}\left(t=1, \hat{x}_{1}, \ldots, \hat{x}_{n}\right)=\prod_{i \in \mathbf{n}} \hat{x}_{i} \sum_{I \subseteq \mathbf{n}}(-1)^{|I|} \operatorname{per} \mathbf{A}^{(\lambda)}(I \mid I) \operatorname{det} \mathbf{A}^{(\lambda)}(\bar{I} \mid \bar{I})=0$.

Theorem 2 and (27) lead to the result

$$
H_{c}=\frac{1}{2 n} \sum_{I \subseteq \mathbf{n}}(-1)^{n-|I|}|I| \operatorname{per} \mathbf{A}^{(\lambda)}(\bar{I} \mid \bar{I}) \operatorname{det} \mathbf{A}^{(\lambda)}(I \mid I) .
$$

To enumerate a permanent, we use Ryser's formula [5]

$$
\operatorname{per} \mathbf{A}^{(\lambda)}(\bar{I} \mid \bar{I})=\sum_{J \subseteq I}(-1)^{|J|} \prod_{j \in J} d_{j, J} \prod_{i \in I-J}\left(d_{i, J}+\lambda_{i}\right),
$$

where

$$
d_{i, I}=\sum_{j \in I} a_{i j}
$$

Note that (16) yields

$$
D\left(t_{1}, \ldots, t_{n}\right)=\sum_{I \subseteq \mathbf{n}}(-1)^{n-|I|}|I| \prod_{i \in I}\left(D_{i}+\lambda_{i} t_{i}\right) \prod_{j \in I} t_{j} \operatorname{det} \mathbf{A}^{(\lambda)}(I \mid I) .
$$


By (28), (29), and (31), we have

\section{Theorem 4.}

$$
H_{c}=\frac{1}{2 n} \sum_{l=0}^{n}(-1)^{l} \Delta_{l},
$$

where

$$
\Delta_{l}=\left.\sum_{I_{l} \subseteq \mathbf{n}} D\left(t_{1}, \ldots, t_{n}\right)\right|_{t_{i}=\left\{\begin{array}{ll}
0, & \text { if } i \in I_{l} \\
1, & \text { otherwise }
\end{array}, \quad i=1, \ldots, n .\right.}
$$

Similarly, we have the asymmetrical case:

\section{Theorem 5.}

$$
H_{c}=\frac{1}{2} \sum_{l=0}^{n-1}(-1)^{l} \Delta_{l}^{(n)},
$$

where

$\Delta_{l}^{(n)}=$

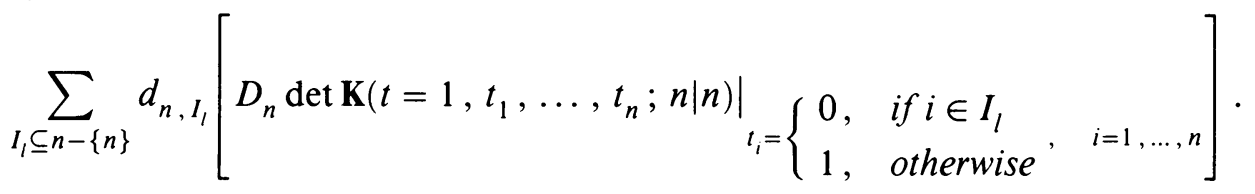

It is interesting to note that $\Delta_{l}$ of (33) has the structural properties of a graph. Let $G_{I}$ be the subgraph obtained from $G$ by deleting all vertices $i \in I$. It is straightforward to show that

\section{Theorem 6.}

$$
\Delta_{l}=\sum_{I_{l} \subseteq \mathbf{n}} \prod_{i \in I_{l}} d_{i, I_{l}} \sum_{j \in \bar{I}_{l}} d_{j, I_{l}} T_{I_{l}}
$$

where

$$
d_{i, I}=(\text { degree of vertex } i \text { in } G)-\left(\text { degree of vertex } i \text { in } G_{I}\right)
$$

and

$$
T_{I}=\text { number of spanning trees in } G_{I} \text {. }
$$

Similarly, $\Delta_{l}^{(n)}$ of (37) may be written

\section{Theorem 7.}

$$
\Delta_{l}^{(n)}=\sum_{I_{l} \subseteq \mathbf{n}} d_{n, I_{l}} \prod_{i \in I_{l}} d_{i, I_{l}} T_{I_{l}}
$$


We now encounter enumeration of Hamiltonian paths in $G$. Let $G^{+}$be the graph obtained from $G$ by adding an $(n+1)$ th vertex together with an edge to each vertex of $G$. It is readily seen that each Hamiltonian path in $G$ can be converted to one and only one Hamiltonian cycle in $G^{+}$; therefore we have

Theorem 8. Let $H_{p}$ be the number of Hamiltonian paths of a given graph. Then

$$
H_{p} \text { in } G=H_{c} \text { in } G^{+} \text {. }
$$

Application. We consider here the applications of Theorem 6 and 7 to a complete multipartite graph $K_{n_{1} \cdots n_{p}}$. It can be shown that the number of spanning trees of $K_{n_{1} \cdots n_{p}}$ may be written

$$
T=n^{p-2} \prod_{i=1}^{p}\left(n-n_{i}\right)^{n_{i}-1}
$$

where

$$
n=n_{1}+\cdots+n_{p}
$$

It follows from Theorems 6 and 7 that

$$
\begin{aligned}
H_{c}= & \frac{1}{2 n} \sum_{l=0}^{n}(-1)^{l}(n-l)^{p-2} \sum_{l_{1}+\cdots+l_{p}=l} \prod_{i=1}^{p}\left(\begin{array}{l}
n_{i} \\
l_{i}
\end{array}\right) \\
& \times\left[(n-l)-\left(n_{i}-l_{i}\right)\right]^{n_{i}-l_{i}} \cdot\left[(n-l)^{2}-\sum_{j=1}^{p}\left(n_{i}-l_{j}\right)^{2}\right]
\end{aligned}
$$

and

$$
\begin{aligned}
H_{c}= & \frac{1}{2} \sum_{l=0}^{n-1}(-1)^{l}(n-l)^{p-2} \sum_{l_{1}+\cdots+l_{p}=l} \prod_{i=1}^{p}\left(\begin{array}{l}
n_{i} \\
l_{i}
\end{array}\right) \\
& \times\left[(n-l)-\left(n_{i}-l_{i}\right)\right]^{n_{i}-l_{i}}\left(1-\frac{l_{p}}{n_{p}}\right)\left[(n-l)-\left(n_{p}-l_{p}\right)\right] .
\end{aligned}
$$

The enumeration of $H_{c}$ in a $K_{n_{1} \cdots n_{p}}$ graph can also be carried out by Theorem 2 or 3 together with the algebraic method of (2). Some elegant representations may be obtained. For example, $H_{c}$ in a $K_{n_{1} n_{2} n_{3}}$ graph may be written

$$
\begin{aligned}
(45) H_{c}=\frac{n_{1} ! n_{2} ! n_{3} !}{n_{1}+n_{2}+n_{3}} \sum_{i}\left[\left(\begin{array}{c}
n_{1} \\
i
\end{array}\right)\left(\begin{array}{c}
n_{2} \\
n_{3}-n_{1}+i
\end{array}\right)\left(\begin{array}{c}
n_{3} \\
n_{3}-n_{2}+i
\end{array}\right)\right. \\
\left.+\left(\begin{array}{c}
n_{1}-1 \\
i
\end{array}\right)\left(\begin{array}{c}
n_{2}-1 \\
n_{3}-n_{1}+i
\end{array}\right)\left(\begin{array}{c}
n_{3}-1 \\
n_{3}-n_{2}+i
\end{array}\right)\right] .
\end{aligned}
$$

\section{ACKNOWLEDGMENT}

It is a pleasure to thank the referee for his valuable suggestions which resulted in an improvement of the manuscript. This paper is dedicated to the memory of my parents. 


\section{REFERENCES}

1. C. J. Liu and Yutze Chow, On operator and formal sum methods for graph enumeration problems, SIAM J. Algebraic Discrete Methods 5 (1984), 384.

2. F. Harary and E. M. Palmer, Graphical enumeration, Academic Press, 1973, p. 226.

3. I. P. Goulden and D. M. Jackson, The enumeration of directed closed Euler trails and directed Hamiltonian circuits by Langrangian methods, European J. Combin. 2 (1981), 131.

4. M. Marcus and H. Ming, A survey of matrix theory and matrix inequalities, Complementary Series in Math. 14 (1964), 21.

5. H. J. Ryser, Matrices with integer elements in combinatorial investigations, Amer. J. Math. 74 (1952), 769.

Department of Mathematics, Statistics, and Computer Science, Marquette UniVersity, MilwaukeE, Wisconsin 53233 